\title{
DIABETES
}

\section{Functional impairment of bone marrow progenitor cells in diabetes mellitus}

A new study published in Diabetes reveals that subpopulations of bone marrow progenitor cells that are important for tissue repair and post-ischaemia recovery are irreversibly impaired in a mouse model of diabetes mellitus.

Researchers from Stanford University, USA, previously identified a subset of progenitor cells in the bone marrow that support vasculogenesis and mobilize into circulation in response to peripheral tissue ischaemia. Reduced levels of circulating progenitor cells have been reported in patients with diabetes mellitus, which suggests that deficiency of these cells could contribute to the pathogenesis of complications associated with this disease.

The scientists demonstrated impaired vasculogenic function of diabetic bonemarrow-derived mesenchymal progenitor cells (BM-MPCs) in vivo and in vitro -an effect that was not reversed when glucose homeostasis levels in the mice were normalized, suggesting that these cells retained irreversible 'diabetic memory'. Using a novel technique that combined single-cell transcriptional analysis and machine learning, the scientists also identified two distinct populations of BM-MPCs that were depleted in diabetic mice compared with wild-type mice. These subsets expressed high levels of vasculogenesis-related genes, which suggests that the depletion of these cells might be involved in impairment of neovascularization in diabetes mellitus.

"Our findings provide the basis for a rational framework to choose and develop cell-based therapies for diabetes mellitus and its complications," says Geoffrey Gurtner, lead author of the study.

Jennifer Sargent

Original article Januszyk, M. et al. Diabetes irreversibly depletes bone marrow-derived mesenchymal progenitor cell subpopulations. Diabetes doi:10.2337/db13-1366 